DOI: https://doi.org/10.46296/yc.v4i7edesp.0059

\title{
CALIDAD DEL PORTAFOLIO DE SERVICIOS DE LA POLICÍA NACIONAL DEL DISTRITO JIPIJAPA
}

\section{QUALITY OF THE PORTFOLIO SERVICES JIPIJAPA DISTRICT NATIONAL POLICE}

\author{
Acebo-Pisco Rosa Judith ${ }^{1 *}$; Anchundia-Loor Andrés Miguel ${ }^{2}$ \\ ${ }^{1}$ Universidad Técnica de Manabí, UTM - Instituto de Posgrado. Portoviejo, Ecuador. \\ ${ }^{2}$ Docente investigador Universidad Técnica de Manabí, UTM. Portoviejo, Ecuador. \\ ${ }^{*}$ Correo: rosaacebop@gmail.com
}

\begin{abstract}
Resumen
Este estudio se centró en analizar el portafolio de servicios que brinda la Policía Nacional ya que es el que posibilita al talento humano, liderando todos aquellos procesos comunitarios y desarrollando acciones preventivas, operativas y estratégicas conformadas por una serie de fortalezas en beneficio de la comunidad, cada uno de los servicios presentan sus ventajas, ya que son programas piloto a incrementarse en la medida que la ciudadanía tenga una alta receptividad, permitiendo aplicar una metodología de tipo transversal no experimental, descriptiva que permiten determinar la calidad del servicio policial del distrito Jipijapa, llevando a cabo cada etapa a la práctica conforme la realidad de cada sector, para efectividad de la mejora se efectuó la adopción de las normas ISO 9001; 2015 ya que estas consisten en organizar un sistema o procedimientos de calidad, reorganizar la institución acogiendo las normativas de calidad y sobre todo planificar una estrategia de mejora que representan como una institución crea y entrega valor a la ciudadanía constituyendo su trabajo y contribuyendo a la mejora de la calidad de servicios acorde a los requerimientos ciudadanos, de donde se concluye que al aplicar esta norma de estándar internacional la organización contará con viabilidad en tiempo y talento humano requerido para lograr los objetivos planteados.
\end{abstract}

Palabras clave: Satisfacción al usuario, policía nacional, ISO 9001, talento humano.

\begin{abstract}
This study focused on analyzing the portfolio of services provided by the National Police since it is one that enables human talent, leading all those community processes and developing preventive, operational and strategic actions made up of a series of strengths for the benefit of the community, Each of the services has its advantages, since they are pilot programs to be increased to the extent that citizens have a high receptivity, allowing the application of a methodology to determine the police service model, carrying out each stage in practice according to reality. For each sector, for the effectiveness of the improvement, the adoption of ISO 9001 standards was carried out; 2015 since these consist of organizing a quality system or procedures, reorganizing the institution accepting quality regulations and above all planning an improvement strategy that represents how an institution creates and delivers value to citizens constituting their work and contributing to improvement of the quality of services according to citizen requirements, therefore this study has the feasibility in time, economic and human talent required to achieve the stated objectives.
\end{abstract}

Keywords: User satisfaction, national police, ISO 9001, human talent.

Información del manuscrito:

Fecha de recepción: 09 de septiembre de 2020.

Fecha de aceptación: 11 de noviembre de 2020.

Fecha de publicación: 16 de noviembre de 2020. 


\section{Introducción}

La calidad es una característica constante que cubre todos los aspectos del trabajo de una organización, por lo que se aplica al desempeño de las personas, incluidas las decisiones y acciones que son esenciales para su nivel de trabajo. De esta forma, se clasifica como una función permanente en la organización de servicios donde los colaboradores son quienes determinan si se cumple la función de calidad y el cliente es quien aprueba si percibe o no dicha calidad en los bienes o servicios que recibe.

Tal como lo explica, Numpaque y Rocha, (2015) "la calidad en los servicios requiere de la aplicación de técnicas que impliquen la prestación oportuna, eficiente, segura $y$ adecuada en cuanto a condiciones físicas y éticas del talento humano, entorno, avances tecnológicos y desarrollos en gestión de las organizaciones" (p.715). Es decir, que se necesita de la aplicación de tecnología y capacitación técnica para que los servicios sean brindados de manera oportuna, eficiente, segura y adecuada en función del estado físico y del ambiente acorde a la capacidad de la gestión organizativa institucional.

En este caso, el portafolio de servicios de la Policía Nacional del Ecuador está distribuido en tres principales tipos que son: servicios estratégicos, servicios operativos y servicios ocasionales, el primero que reúne los servicios de asamblea comunitaria, contacto ciudadano, escuela segura, botón seguro, capacitación a la ciudadanía, espacio público, local seguro y barrio seguro; en el segundo se comprenden los servicios de atención ciudadana, auxilio y respuesta, así como reacción y disuasión, es decir, patrullaje vehicular de apoyo; mientras que el tercero comprende el traslado de valores, encargo domiciliario y guía de movilización; si bien es cierto, este amplio paquete de servicios que brinda la Policía Nacional como institución de orden gubernamental en beneficio de la sociedad ecuatoriana, constituye un abanico muy diverso de servicios que por ende, su ejecución debe de bridarse bajo estándares de calidad que el usuario sea capaz de percibir en su pleno goce de los derechos civiles que la ley le faculta y que como ciudadano nacional tiene derecho a 
acceder a un servicio de excelencia

humana,

organizacional $\quad y$

tecnológica.

Este artículo se centra en un enfoque de gestión de la calidad sistemático y con visión de futuro, al tiempo que incorpora principios y conceptos clave que están cada vez más en consonancia con la competitividad actual. Según, Bernedo, (2017) explica que "la cultura organizacional y la calidad de servicios que brinda una entidad de seguridad a la comunidad está claramente relacionada a los métodos con la que se brindan los servicios a los ciudadanos que esperan confianza, amabilidad y respeto" (p.265). En este sentido, es evidente que la institución necesita delinear una cultura organizacional donde los agentes de policía establezcan buenas relaciones y fortalezcan la práctica de las relaciones interpersonales, así como el trabajo en equipo y liderazgo participativo para buscar la satisfacción del usuario acorde a la visión de la organización.

\subsection{Gestión de la calidad en las organizaciones.}

Según, la norma internacional, ISO
9001 (2015) establece los requisitos de los Sistemas de Gestión de la calidad en las organizaciones, aplicables a todo tipo de organizaciones sean públicas 0 privadas, así como productoras de bienes o servicios, donde establece que entre los principios de la gestión de la calidad, se encuentran aspectos tales como: el enfoque al cliente, el liderazgo organizacional, el compromiso de las personas 0 colaboradores, el enfoque a procesos, la mejora contínua, la toma de decisiones basada en la evidencia, y la gestión de las relaciones.

Es decir, que esta norma internacional aboga por el uso de métodos de proceso al desarrollar, implementar y mejorar la efectividad del sistema de gestión de la calidad para mejorar la satisfacción del cliente al cumplir con los requisitos del cliente.

Es por esto que resulta conveniente para una institución tan seria como la Policía Nacional, comprender y gestionar los procesos interrelacionados como un sistema ayuda a la organización a lograr la eficacia y eficiencia de los resultados esperados, mediante la aplicación de 
modelos de gestión que le permitan controlar las interrelaciones e interdependencias entre los procesos del sistema, lo que dará como resultado el mejoramiento del desempeño general de la organización y con ello la satisfacción plena de sus usuarios que en efecto es la sociedad en general.

Si bien, el enfoque de procesos de cada uno de los servicios que ofrece la entidad significa la definición y gestión sistemática de los procesos y sus interacciones con el fin de obtener los resultados esperados de acuerdo con la política de calidad y la dirección estratégica de la organización, apoyado en el ciclo PDCA y el enfoque global del pensamiento orientado al riesgo para gestionar procesos y todo el sistema para aprovechar oportunidades y prevenir consecuencias adversas.

\subsection{Calidad en los servicios}

A propósito, Moreno (2013) sostiene que "hoy en día, la calidad y seguridad en los servicios se ha convertido en una de las principales prioridades a nivel mundial y en todos los niveles" (p.18). Esto indica que, cada vez es las organizaciones tanto públicas como privadas tienen la obligación o la responsabilidad de asegurar que la atención que brindan a sus usuarios cumpla con los estándares internacionales de calidad y protegerlos de los riesgos inherentes a la gestión organizacional es un desafío que requiere el compromiso de todo el personal relevante de la organización.

Para lograr el reto de la calidad en una organización cuya misión es atender la seguridad ciudadana y el orden público, así como proteger el libre ejercicio de los derechos y la seguridad de las personas dentro del territorio nacional, se necesita un compromiso institucional, multidisciplinario e interdisciplinario, pero la organización no lo es todo, también se necesita de la colaboración en este proceso de parte del usuario receptor de la atención, es decir que la contribución de todos y cada uno de los actores sociales involucrados en el ámbito de la seguridad ciudadana permitirá garantizar que una atención que cumpla con los estándares de calidad y minimice riesgos para la ciudadanía a nivel local, nacional e internacional; no obstante, las 
diferencias en la calidad y seguridad que se proporciona en las diversas instituciones siguen siendo notorias, se requiere un mayor esfuerzo para afirmar que la atención que se proporciona a todo usuario independientemente del tipo de institución a la que acuda a solicitar atención, es de calidad y libre de riesgo.

\subsection{Evaluación de la calidad en los servicios}

Según, Tudela (2015) explica que "la calidad de los servicios policiales y los modelos de gestión policial son claves en la seguridad pública y ciudadana y, por lo mismo, en la calidad de la democracia" (p.3). Es decir, la calidad de los servicios policiales este es un gran desafío porque implica avanzar en la implementación de políticas de prevención y control, asegurando un buen desempeño policial cuando se considere innecesario; en efecto, este desafío solo se puede enfrentar a través de la evaluación de resultados que significan modificaciones en el clima laboral, fortalecimiento de los conocimientos, actitudes, satisfacción, nivel de habilidad y grado de cumplimiento del tratamiento al ciudadano considerando criterios tales como: la responsabilidad para comprender la necesidad de atención; el nivel de información, comunicación, interés y cortesía; la rapidez en la atención; la mejora continua; y el modo en que ofrezca los servicios tanto administrativos, de seguridad, entre otros.

Actualmente, la evaluación es fundamental para la formulación efectiva de políticas, pues la estrategia policial debe ser revisada y confirmada, y debe prestarse atención a los diferentes indicadores que pueden ser utilizados por la policía y los organismos encargados de la prevención y control del delito o actuación. Del mismo modo, se deben colmar las brechas de información en las políticas y la seguridad públicas, por ejemplo, estudiando hasta qué punto las estrategias policiales son efectivas para reducir el miedo y el número de delincuentes.

\subsection{Gestión de la calidad de los servicios}

El Sistema de Gestión de Calidad (SGC) de la Policía Nacional del Ecuador está abocado como un requisito en la implementación del 
Sistema de Gestión Integrado (SGI) para el diseño e implantación de un sistema propio de gestión de la calidad, de acuerdo con los lineamientos de las normas ISO 9001:2015 porque es un modelo muy adecuado para organizaciones que deben continuar brindando servicios complejos en todo el país (Nacional, 2016).

En este sentido, los cambios institucionales requieren que la policía nacional desarrolle e implemente estándares de capacitación e implementación para reflejar la especialización procesal efectiva de la organización, estructura y recursos, así como conocimiento y desempeño del personal a fin de asegurar el cumplimiento de los estándares mínimos y la formación necesaria tanto a nivel organizacional como a nivel de desempeño individual.

\subsection{Estándares de personal}

El estándar del personal policial de atención en las unidades de policía comunitaria, obedece a una lógica operativa en la cual se desea mantener las Unidades de Policía Comunitaria (UPC) con policías en turnos de 8 horas de trabajo, cubriendo tres servicios básicos para la ciudadanía, que son: Policías en funciones de Policía Comunitaria, acciones permanentes de acercamiento comunitario (POLCO), patrullaje vehicular permanente $y$ patrullaje motorizado continuo, en acciones de prevención y respuesta (Policía Nacional del Ecuador, s/f).

La asignación de cada tipo de número personal estándar asegura que los servicios se presten en cada una de las UPC las 24 horas del día, los 365 días del año, los francos correspondientes que deben tener los agentes de policía y las funciones a desempeñar.

\subsection{Portafolios de servicios}

Según, Flórez, Ramírez y Quintero (2020) "el porfolio de servicios constituye una herramienta para que las organizaciones den a conocer a sus clientes o usuarios todo el trabajo que realizan u ofertan" (p.5).

En este sentido, los servicios policiales proporcionados a nivel de subcircuito son actividades, procesos, procedimientos diseñados para cumplir con los siguientes requisitos La seguridad necesita una convivencia pacífica con los ciudadanos.

Si bien, el portafolio de servicios de 
la Policía Nacional se ha agrupado en tres servicios: Los servicios estratégicos, servicios operativos y servicios ocasionales, todos estos servicios darán respuesta a los requerimientos y necesidades de los ciudadanos del sector o grupos de interés específicos mediante estrategias que se proponen a la comunidad tales como:

Gestión policial comunitaria para prevención del delito y proximidad con la ciudadanía (estratégicos)

- Desarrollo de Asamblea Comunitaria.

- Capacitación a la ciudadanía

- Chat comunitario.

- Espacio público recuperado.

- Gestión para implementación de cámaras de video vigilancia.

- Sensibilización a la comunidad.

- Actividades lúdicas.

- Coordinación de Campamentos Vacacionales

- Minutos Cívicos.

- Ferias de seguridad.

- Visitas preventivas comunitarias.

- Escuelas de seguridad ciudadana.
- Contacto ciudadano post delito.

- Activación botón de Seguridad.

- Aplicaciones tecnológicas interactivas comunitarias.

Servicio de atención y patrullaje policial (operativos)

- Atención ciudadana.

- Patrullaje vehicular.

Atención de requerimientos ocasionales (ocasionales)

- Visitas periódicas a domicilio

- Emisión de guía de movilización

- Custodia policial de bienes y valores

En conclusión, el portafolio servicios policial contiene una serie de actividades, procesos y procedimientos destinados a satisfacer las necesidades de los ciudadanos para una convivencia segura y pacífica, que cumplen con los requerimientos y necesidades de los ciudadanos del sector o grupos de interés específicos.

\section{Metodología (Materiales y métodos)}

El presente trabajo de investigación de tipo transversal no experimental, 
descriptiva, que busca determinar calidad del portafolio de servicios de la Policía Nacional del Distrito Jipijapa, además presenta un enfoque cuantitativo por medio de la aplicación de encuestas a los usuarios de dichos servicios, para ello se determinó una muestra representativa de personas que fueron encuestadas partiendo de la población universo, objeto de estudio de esta investigación. El estudio se efectúo en la ciudad de Jipijapa incluyendo a personas de la sociedad civil o ciudadanos comunes que están facultados para demandar dicho servicio, a la que se aplicó técnicas e instrumentos que permitieron conocer la percepción del usuario respecto a la calidad. Además, la investigación se sustentó en una revisión bibliográficadocumental, con la finalidad de definir conceptual y científicamente las variables de investigación.

\subsection{Población}

La población considerada para el presente estudio está sustentada en los datos del INEC (2010) que señalaba 40200 habitantes, con una tasa de crecimiento poblacional $+1.22 \%$ /Año por lo que al 2020 se estima una población cantonal de 45
411 habitantes, quienes sin importar su edad pueden ser usuarios directos o indirectos de los servicios que presta la Policía Nacional en el Distrito Jipijapa.

\subsection{Muestra}

Según la población definida en el presente estudio, para analizar la satisfacción de los usuarios de los servicios policiales de sus habitantes, corresponde aplicar la técnica de muestreo, tal como se enuncia en la siguiente expresión estadística:

\begin{tabular}{|c|c|c|c|c|c|c|c|c|c|}
\hline \multirow{2}{*}{$n=$} & $(Z)^{2}\left(P^{*} Q\right)(N)$ & & & & & & & & \\
\hline & $(1)(N)+(Z)^{2}\left(P^{*} Q\right)$ & & & & & & & & \\
\hline & Donde: & \multirow{2}{*}{$n=$} & $(1,96)$ & 2 & \multicolumn{2}{|c|}{$(0,25)$} & & & (45.411) \\
\hline $\mathrm{n}=$ & Tamaño de la muestra & & $(0,05)$ & 2 & \multicolumn{4}{|c|}{$(45.411)+(1,96)^{2}$} & $(0,25)$ \\
\hline$Z=$ & Margen de confiabilidad, & \multirow{3}{*}{$n=$} & \multirow{2}{*}{\multicolumn{2}{|c|}{$(3,84)$}} & \multirow{2}{*}{\multicolumn{2}{|c|}{$(0,25)$}} & & \multirow{2}{*}{\multicolumn{2}{|c|}{ (45.411) }} \\
\hline$e=$ & Error admisible & & & & & & & & \\
\hline \multirow[t]{2}{*}{$\mathrm{N}=$} & Tamaño de la población & & \multicolumn{2}{|c|}{$(0,0025)$} & \multicolumn{2}{|c|}{$(45.411)$} & \multicolumn{3}{|c|}{$+(3,84)(0,25)$} \\
\hline & & \multirow{2}{*}{$n=$} & \multicolumn{3}{|c|}{43.613} & & & & \\
\hline \multicolumn{2}{|c|}{$\mathrm{n}=?$} & & 113,53 & $+c$ & 0,96 & & & & \\
\hline \multicolumn{2}{|c|}{$Z=1,96$} & \multirow{3}{*}{$n=$} & & & & & & & \\
\hline$P=$ & 0,5 & & 43.613 & & & & & & \\
\hline$Q=$ & 0,5 & & 114,49 & & & & & & \\
\hline \multicolumn{2}{|c|}{$e=0,05$} & & & \multirow{3}{*}{\multicolumn{3}{|c|}{ Encuestados }} & & & \\
\hline$N=$ & 45.411 & $n=$ & $381 \mathrm{E}$ & & & & & & \\
\hline$Z=$ & 0,95 & & & & & & & & \\
\hline
\end{tabular}

\section{Resultados (análisis e interpretación de los resultados)}

Luego del despliegue investigativo en el presente trabajo se presentan los siguientes resultados:

La ciudadanía del cantón Jipijapa es usuaria de los tres principales tipos de servicios que brinda la Policía 
Nacional en su portafolio dirigido a la comunidad, siendo los servicios estratégicos los más demandados por los beneficiarios, siendo mayormente demandado aquellos servicios estratégicos y en menor escala aquellos servicios operacionales y ocasionales, tal como se muestra en la tabla No. 1.

Tabla No. 1. Servicios de la Policía Nacional demandados en el distrito Jipijapa.

\begin{tabular}{lcc}
\hline \multicolumn{1}{c}{ Alternativa } & Frecuencia & $\%$ \\
\hline $\begin{array}{l}\text { Servicios } \\
\text { estratégicos }\end{array}$ & 289 & $76 \%$ \\
$\begin{array}{l}\text { Servicios } \\
\text { ocasionales }\end{array}$ & 43 & $11 \%$ \\
$\begin{array}{l}\text { Servicios } \\
\text { operacionales }\end{array}$ & 49 & $13 \%$ \\
Total & $\mathbf{3 8 1}$ & $\mathbf{1 0 0}$ \\
\hline
\end{tabular}

Fuente: Usuarios del Distrito Jipijapa. Elaborado por: Autores.

De forma más específica, respecto a los servicios estratégicos, se evidenció que la ciudadanía demanda este tipo de servicios en un $76 \%$ a nivel distrital, en sus respectivos sub-servicios, tal como se muestra en el siguiente gráfico.
Figura No. 1. Demanda de servicios estratégicos.

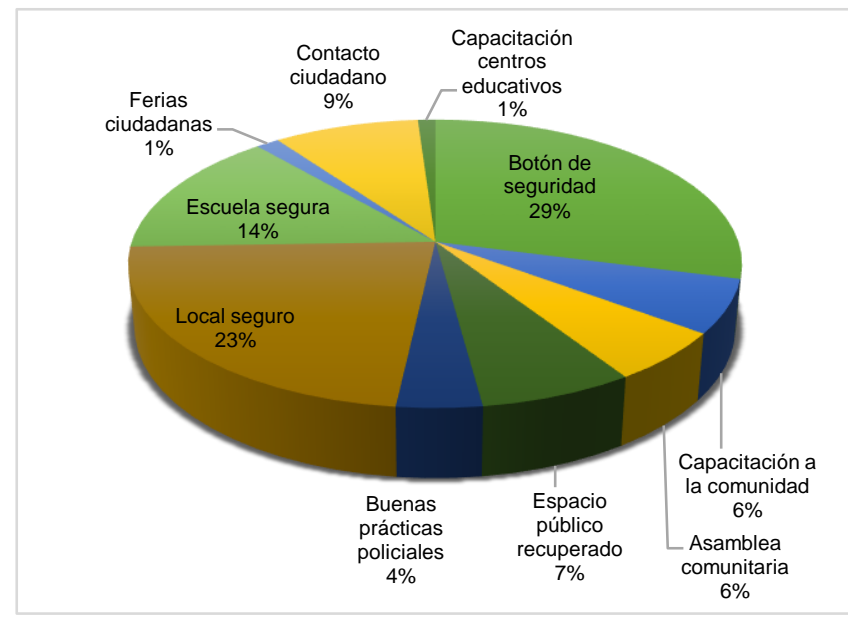

Fuente: Usuarios del Distrito Jipijapa. Elaborado por: Autores.

Otro tipo de servicios contenido en el portafolio de servicios de la Policía Nacional y que son solicitados por la ciudadanía son los servicios ocasionales, que representan el $11 \%$ de la demanda global, entre los cuales se detallan los siguientes:

Figura No. 2: Demanda de servicios ocasionales.

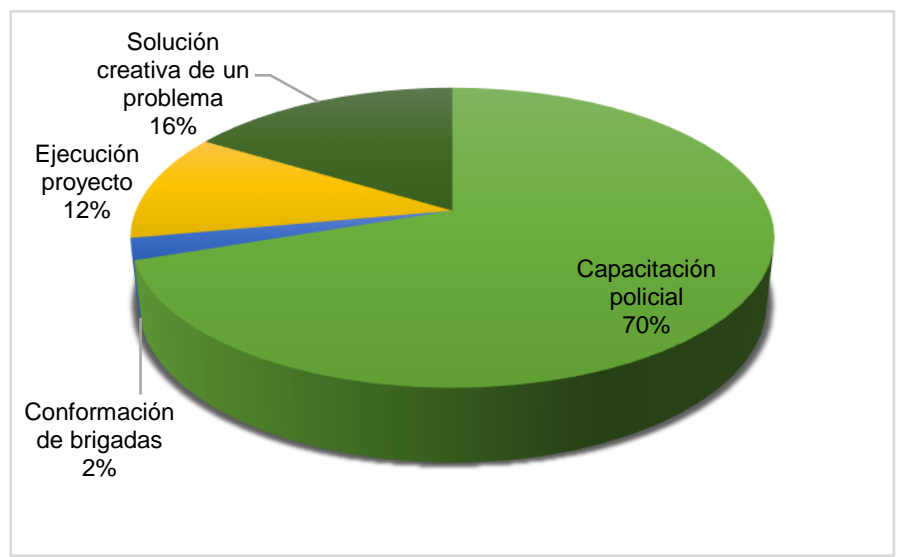

Fuente: Usuarios del Distrito Jipijapa. Elaborado por: Autores. 
Además, la sociedad Jipijapense solicita servicios operacionales que representan el $13 \%$ de la demanda global de servicios policiales en la ciudad, referidos a los siguientes:

Figura No. 3. Demanda de servicios operacionales.

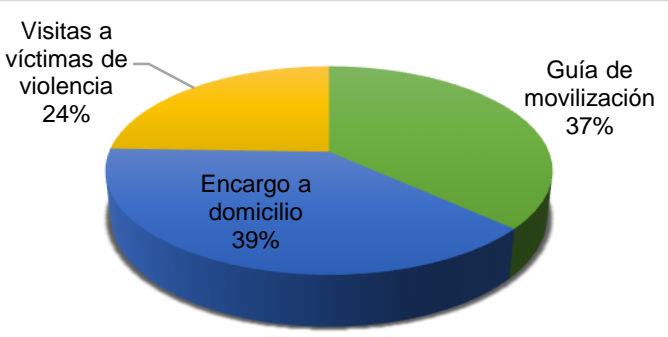

Fuente: Usuarios del Distrito Jipijapa. Elaborado por: Autores.

De todos estos tipos de servicios ofrecidos por la Policía Nacional, se evidencia su demanda totalitaria de los mismos, en efecto, unos son más demandados que otros, los cuales, en términos de calidad, han sido evaluados por los usuarios de la siguiente manera:

Figura No. 4. Evaluación de la calidad de los servicios de la Policía Nacional.

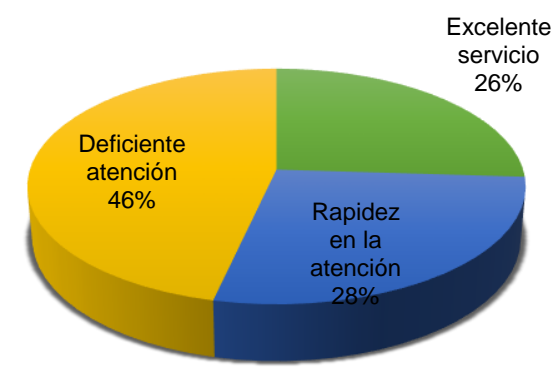

Fuente: Usuarios del Distrito Jipijapa. Elaborado por: Autores.
Tal como se evidencia en la figura No. 4 respecto a la evaluación de la calidad de los servicios de la Policía Nacional, la población considera en un $46 \%$ que existe deficiencia en el servicio, el $28 \%$ considera que éstos son rápidos por su atención y el $26 \%$ lo considera como excelente.

En base a lo expuesto anteriormente, se evidencia la necesidad de someter a un análisis de la calidad a cada uno de los servicios que ofrece la organización a fin de mejorar continuamente $y$ satisfacer la demanda de los usuarios como la consecución de los propósitos misionales de la institución.

\section{Conclusiones}

El portafolio de servicios que ofrece la Policía Nacional en el Distrito Jipijapa es amplio y su demanda es muy variada, en dichos servicios se comprenden actividades, procesos y procedimientos destinados a cumplir con requisitos de seguridad $y$ convivencia pacífica con los ciudadanos, por lo que en pro de la calidad organizacional los agentes reciben constante capacitación en derechos humanos, investigaciones especializadas, control y prevención 
del delito, así como métodos de persuasión y reconciliación para que el cumplimiento de sus funciones sea lo más efectiva en todos los niveles.

Los servicios más demandados por la ciudadanía del Distrito Jipijapa de la Policía Nacional son los de tipo estratégicos, específicamente aquellos relacionados al botón de seguridad, local seguro y escuela segura, mientras que respecto a los servicios ocasionales la ciudadanía demanda mayoritariamente los servicios de capacitación policial, y referente a los servicios operacionales se demandan servicios de guía de movilización, lo que supone para la institución el fortalecimiento de este tipo de servicios a fin de garantizar la seguridad ciudadana, es decir, está destinada a modernizar los mecanismos necesarios para garantizar los derechos humanos, en especial el derecho a una vida libre de violencia y criminalidad, la disminución de los niveles de delincuencia, la protección de víctimas y el mejoramiento de la calidad de vida de todos los habitantes.

En referencia a la calidad percibida por los usuarios, ésta se traduce en una calificación como "deficiente", por ende, supone para la institución un reto de medidas preventivas y de servicio a la ciudadanía, registro y acceso a información, la ejecución de programas ciudadanos de prevención del delito y de medición de la calidad en cada uno de los procesos a fin de garantizar servicios de vigilancia y respuesta que beneficien a la institución y a la ciudadanía en general.

\section{Bibliografía}

Bernedo, V. (2017). Incidencia de la cultura organizacional de los efectivos de la región policial Sur en la calidad de servicios de la comunidad de Arequipa - 2015. Universidad Andina. http://repositorio.uancv.edu.p e/bitstream/handle/UANCV/9 07/T036_43369139_D.pdf?se quence $=3 \&$ is Allowed $=y$

Flórez, V., Ramírez, D., \& Quintero, A. (2020). Diseño de portafolio de servicios que sirva para el mejoramiento de la visibilidad de la Fundación Cosmos.

Bucaramanga, Santander.

http://repositorio.uts.edu.co:8 080/xmlui/handle/123456789/ 2944

INEC. (2010). Población por sexo y grupos de edad. 
http://redatam.inec.gob.ec/cgi bin/RpWebEngine.exe/Portal Action?\&MODE=MAIN\&BAS $E=C P V 2010 \& M A I N=$ WebSer verMain.inl

ISO 9001. (2015). Sistemas de Gestión de la calidad. Requisitos (ISO 9001:2015, IDT). ISO.

Moreno, M. (2013). Calidad y seguridad en la atención. Universidad Autónoma de Nuevo León. https://scielo.conicyt.cl/scielo. php?script=sci_arttext\&pid $=$ S 0717-95532013000100001

Nacional, P. (2016). Plan Estratégico 2016-2020. Dirección General de la Policía Nacional. https://www.policianacional.g ob.do/wpcontent/uploads/2017/02/PLA N_ESTRATEGICO_20162020_enero_2017-1.pdf

Numpaque, A., \& Rocha, A. (2015). Modelo SERVQUAL y SERVQHOS para la evaluación de la calidad de los servicios de salud (Vol. 64). Revista de la Facultad de Medicina.

http://www.scielo.org.co/pdf/rf mun/v64n4/0120-0011-rfmun64-04-00715.pdf

Policía Nacional del Ecuador. (s/f). Curso de ascenso de los señores Servidores Policiales Técnicos Operativos.
Tudela, P. (2015). La medición del desempeño policial en Chile: consideraciones sobre la calidad de los servicios y el trato a víctimas $\mathrm{y}$ denunciantes.

https://biblioteca.cejamericas. org/bitstream/handle/2015/847 /policiapercepcion.pdf?sequen $\mathrm{ce}=1$ \& isAllowed $=\mathrm{y}$ 